\title{
Positron trapping in $\mathrm{BaTiO}_{3}$ perovskite
}

To cite this article: C Macchi et al 2001 J. Phys.: Condens. Matter 135717

View the article online for updates and enhancements.

\section{Related content}

\author{
Temperature dependence of positron \\ trapping at grain boundaries \\ S Aina, A Dupasquier, P Folegati et al. \\ On the two-step ageing of a commercial A \\ - Zn - Mg alloy; a study by positron lifetime \\ spectroscopy \\ R Ferragut, A Somoza and A Dupasquier \\ Positron lifetime spectroscopy and \\ decomposition processes in commercial \\ Al-Zn-Mg-based alloys \\ R Ferragut, A Somoza and A Dupasquier
}

\section{Recent citations}
Structure and dielectric properties of lower valence compensation for Ba1-

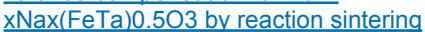 process
Yin-Lai Chai et al
- A study of the structure and dielectric properties for hiqh-valence compensation of $\mathrm{Bi} 3+$ ion-doped $\mathrm{Ba}(\mathrm{FeTa}) 0.5 \mathrm{O} 3$ ceramics
Yin-Lai Chai et al
- Dielectric properties and structural defects in $\mathrm{BaTi}_{4} \mathrm{Sn}_{*} \mathrm{O}_{3}$ ceramics Zhang Lijuan et al

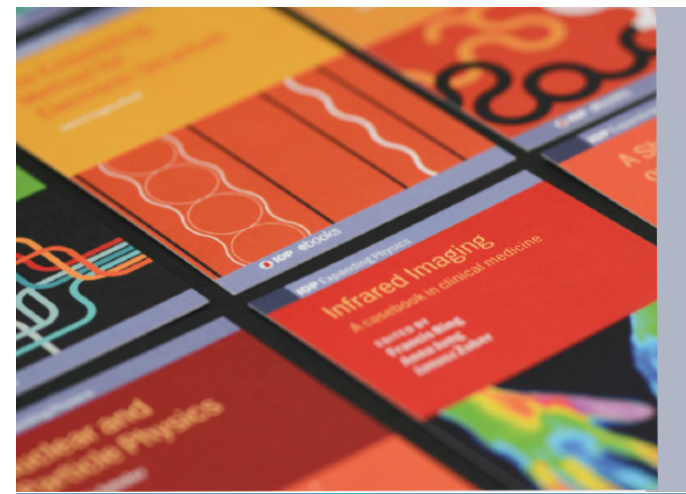

IOP ebooks

Bringing together innovative digital publishing with leading authors from the global scientific community. Start exploring the collection-download the first chapter of every title for free. 


\title{
Positron trapping in $\mathrm{BaTiO}_{3}$ perovskite
}

\author{
C Macchi ${ }^{1}$, A Somoza ${ }^{1,5}$, A Dupasquier ${ }^{2}$, A López García ${ }^{3}$ and M Castro ${ }^{4}$ \\ 1 IFIMAT-UNCentro and Comisión de Investigaciones Científicas de la Provincia de Buenos \\ Aires, Pinto 399, 7000 Tandil, Argentina \\ 2 Istituto Nazionale di Fisica della Materia and Dipartimento di Fisica, Politecnico di Milano, \\ Piazza L da Vinci 32, 20133 Milano, Italy \\ ${ }^{3}$ Departamento de Física, Facultad de Ciencias Exactas, Universidad Nacional de La Plata \\ and Consejo Nacional de Investigaciones Científicas y Técnicas, CC 67, 1900 La Plata, Argentina \\ ${ }^{4}$ INTEMA-Universidad Nacional de Mar del Plata, J B Justo 4302, 7600 Mar del Plata, Argentina \\ E-mail: asomoza@exa.unicen.edu.ar
}

Received 8 January 2001, in final form 11 April 2001

\begin{abstract}
Positron lifetime spectra in $\mathrm{BaTiO}_{3}$ single crystals were measured at temperatures up to $873 \mathrm{~K}$, also at room temperature after quenching from temperatures up to $473 \mathrm{~K}$. The explored temperature range includes the ferroelectric to paraelectric phase transition. The material displays an irreversible behaviour when heated, with anomalies around $T_{C}$. The temperature dependence of the positron lifetime becomes reversible only after annealing above $550 \mathrm{~K}$. The results suggest modifications of the charge state of non-equilibrium vacancies occurring at moderate temperature and microstructural changes taking place at high temperature. The reversible behaviour of the annealed crystal is consistent with thermally activated positron trap formation.
\end{abstract}

Barium titanate $\left(\mathrm{BaTiO}_{3}\right)$ is a text-book example of displacement-type ferroelectric perovskite [1], widely studied for its interesting basic properties and extensive applications. In this material, defects originated by the preparation method and contaminants in the reactants have been observed [2]. As a consequence, cation and anion vacancies as well as impurities at the $\mathrm{Ba}$ and Ti sites can be expected in as-prepared samples. Oxygen vacancies often compensate for metallic impurities. The electrical properties of barium titanate, including ferroelectricity, are strongly influenced by the presence of the above-mentioned defects [3].

Positron annihilation spectroscopy (PAS) is a well established technique for the study of vacancies, due to the sensitivity of annihilation characteristics to the local electron density and to ability of open-volume defects to trap positrons. In particular, the positron lifetime technique (PALS) has been successfully used to study the thermal formation of vacancies in metals and alloys $[4,5]$ and, recently, in intermetallics $[6,7]$. The application of PAS to the study of defects in insulators and semiconductors is also possible, although it is complicated

5 Corresponding author. 
by the modifications of the charge state of the defects occurring with temperature changes and doping [8].

Previous information regarding positron annihilation in $\mathrm{BaTiO}_{3}$ can be found in [9-14]. Positron lifetime effects related to structural phase transitions, tetragonal-orthorhombicrhombohedral, and to the corresponding hystereses were observed in $\mathrm{BaTiO}_{3}$ during temperature dependent measurements in the range of $77 \mathrm{~K}$ to about room temperature [11]. In contrast, angular correlation and lifetime measurements $[9,14]$ in the temperature range including the ferroelectric to paraelectric phase transition gave no evidence of any sensitivity of positron annihilation characteristics to this transition. Calculations for the lifetime for free and defect-trapped positrons were reported by Ghosh et al [15]. In the present work, the evolution of the positron lifetime in single crystals of barium titanate has been measured in situ during thermal cycles including the critical temperature $T_{c}$ of the ferroelectric-paraelectric transition.

The single crystals were prepared by melting $\mathrm{BaTiO}_{3}$ powder in $\mathrm{KF}$ flux and by cooling down the melt at a rate of $10 \mathrm{~K} \mathrm{~h}^{-1}$. The estimated concentration of accidental metallic impurities $(\mathrm{Na}, \mathrm{K}, \mathrm{Fe})$ is of the order of 100 atomic ppm for each species, for a total content of impurities below 1000 atomic ppm. At thermal equilibrium, monovalent impurities at $\mathrm{Ba}$ sites and trivalent impurities at Ti sites are compensated by oxygen vacancies [2]. As-prepared single crystals were transparent with a light yellow tint. The samples used for PALS were cut to a thickness of $1.5 \mathrm{~mm}$ from the single crystals by a low speed diamond saw. The damaged surface was removed by mechanical polishing.

Positron lifetime spectra were measured with a conventional ORTEC fast-fast time spectrometer with a resolution of $250 \mathrm{ps}$ (FWHM). A ${ }^{22} \mathrm{NaCl}$ source of about $35 \mu \mathrm{Ci}$ was directly deposited onto the samples. During a typical measurement, about $10^{6}$ coincidence counts were collected. The spectra were analysed by means of the POSITRONFIT program

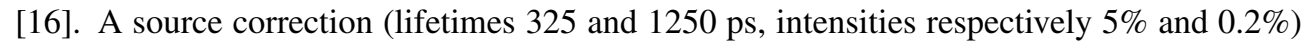
was applied.

The measurements were performed between RT and $873 \mathrm{~K}$. The temperature of the samples was monitored by soldering chromel-alumel thermocouples to the samples, and was automatically stabilized to $\pm 2 \mathrm{~K}$.

The lifetime spectra observed in as-prepared samples could not be analysed in more than one component. The mean life of this component, which however might be an average over an

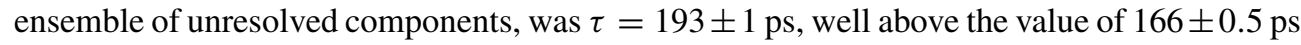
that was obtained after annealing (see below). Figure 1 depicts the evolution of $\tau$ in a thermal cycle reaching the temperature of $473 \mathrm{~K}$. During the heating stage, $\tau$ shows a monotonic decrease, until it reaches a relative minimum of $190 \mathrm{ps}$. This minimum is located very close to the critical temperature $\left(T_{c} \approx 393 \mathrm{~K}\right)$ of the ferroelectric to paraelectric phase transition. A further increase in the measurement temperature induces an increase in the positron lifetime up to $192 \mathrm{ps}$, followed by a marked decrease when the temperature is higher than about $420 \mathrm{~K}$. The lifetime minimum (186 ps) is reached at the highest temperature of the cycle. During the cooling stage, the positron lifetime remains almost constant at this level. Further heating at two temperatures below and above $T_{c}$ did not produce lifetime changes.

The irreversible behaviour shown in figure 1 demonstrates the presence of native lattice disorder in the as-prepared single crystals. Positrons are trapped in vacancy-like defects and annihilate there with a lifetime longer than in the bulk. The presence of a dip around $393 \mathrm{~K}$ in the curve representing the heating stage suggests that at least some of the positron traps can change their charge state from negative or neutral to positive when random internal electric fields relax due to the onset of the paraelectric behaviour. Following this line of interpretation, the part of the heating curve with a positive slope (from about 390 to $410 \mathrm{~K}$ ) might indicate an opposite change of the charge state, due to an upward displacement of the Fermi level. On the other hand, 


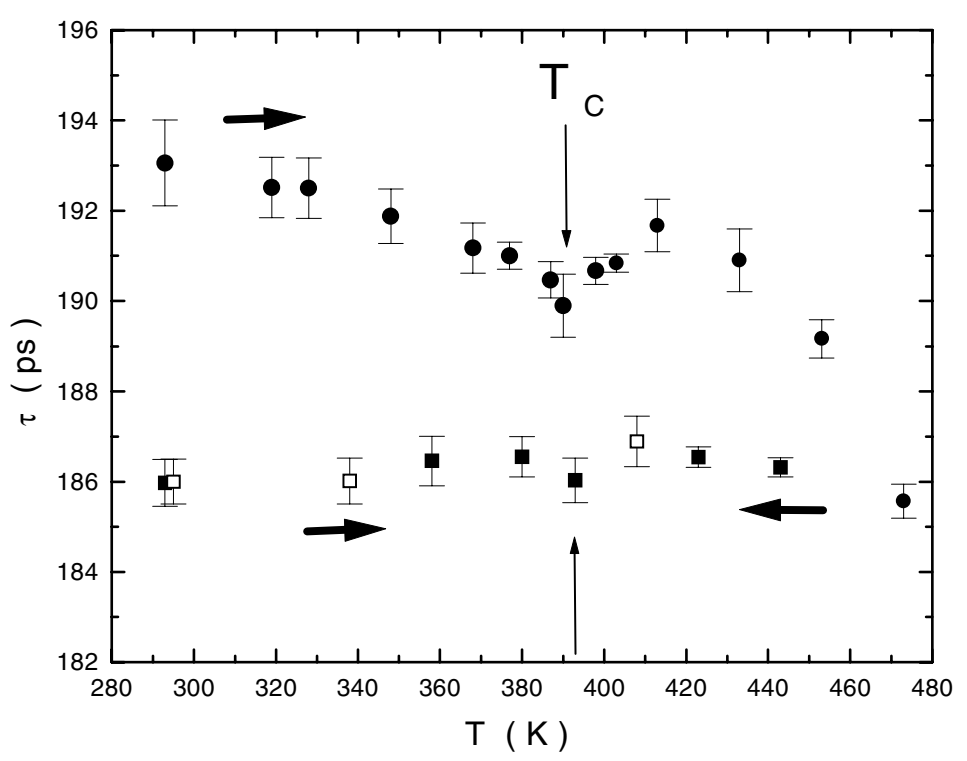

Figure 1. Evolution of the average positron lifetime as a function of the temperature in as-prepared $\mathrm{BaTiO}_{3}$ single crystals: - first heating stage, $\mathbf{\square}$ cooling stage, $\square$ second heating stage. Two overlapping points at $293 \mathrm{~K}$ (cooling stage and second heating stage) have been artificially separated in the figure.

the negative slope of the curve observed above $420 \mathrm{~K}$ is certainly to be seen as an irreversible recovery stage, during which the initial structural disorder is gradually disappearing. When the heating cycle is interrupted at $473 \mathrm{~K}$, the recovery is incomplete, as shown by a positron lifetime still above the minimum observed in well annealed samples. The nature of the residual traps seems to be different from that of the traps that have already disappeared, since the reduced sensitivity of the positron lifetime to the temperature indicates that the dominant traps are in a fixed charge state. Positron trapping at Ba vacancies has been suggested as the main cause of positron trapping in $\mathrm{BaTiO}_{3}$ by Süvegh et al [13]. However, attempts to resolve the lifetime predicted [15] for Ba vacancies (293 ps) by constrained fits of the lifetime spectra were unsuccessful. A superposition of components associated with Ti and $\mathrm{O}$ vacancies (predicted lifetimes 204 ps and 162 ps respectively) [15] would be in better agreement with the experimental results. The effect of oxygen vacancies, possibly in the form of complexes with metal ion vacancies, is also strongly supported by the results of Massoud et al [14]. Oxygen vacancies are believed to be effective positron traps in other perovskites [17-19]. Trapping at oxygen vacancies and vacancy clusters in YBCO has also been predicted by several calculations [20-22]. The non-monotonic temperature dependence of the positron lifetime observed during the heating stage of the thermal cycle might be explained in terms of a change of the charge state of oxygen vacancies from neutral to positive and to neutral again under opposite actions of internal electric fields and temperature on the position of the local Fermi level.

Fully reversible dependency of the annihilation characteristics on the temperature, was obtained after thermal treatments above $550 \mathrm{~K}$ in air. Only one component was resolved at temperatures below $523 \mathrm{~K}$; free fits in two components were successful at temperatures from $523 \mathrm{~K}$ to $773 \mathrm{~K}$. As shown in figure 2 (open diamond), the lifetime $\tau_{2}$ of the second component has a mean value of $219 \pm 3 \mathrm{ps}$, without a systematic temperature dependence. This value, which is intermediate between the predicted values for trapping at $\mathrm{Ti}$ and at $\mathrm{Ba}$ vacancies, 


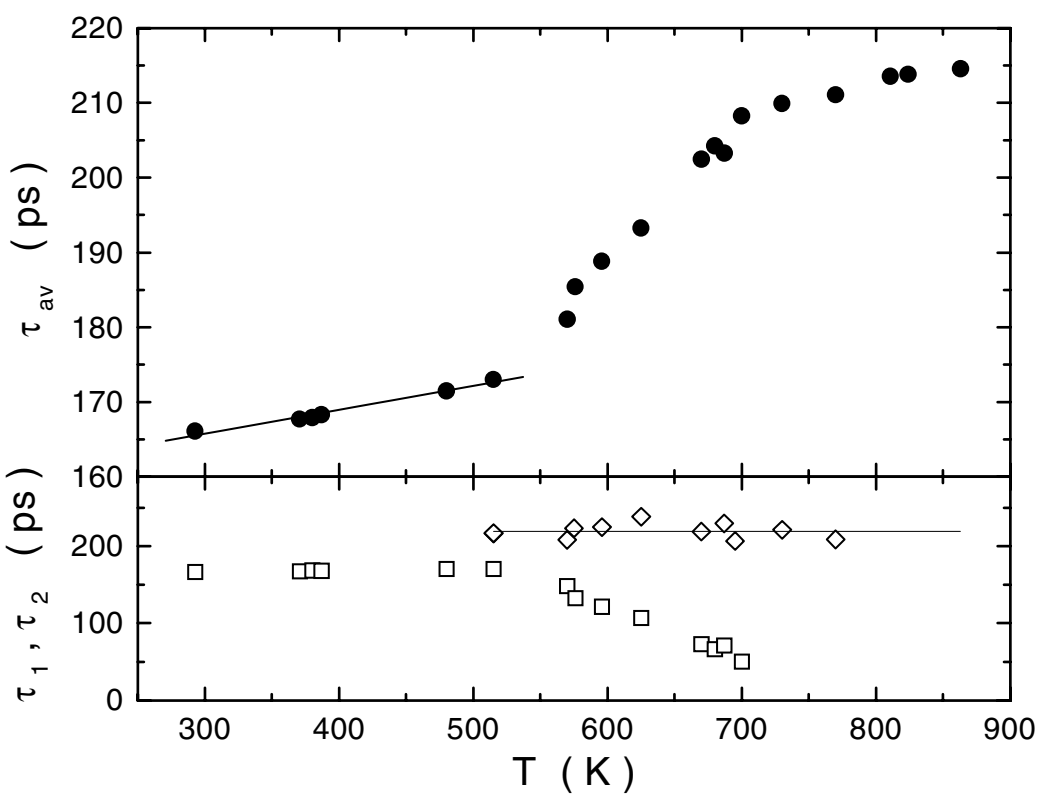

Figure 2. Annihilation characteristics in well annealed $\mathrm{BaTiO}_{3}$ single-crystals: lifetime $\tau_{2}$ of the long living component from free fits (open diamonds); lifetime $\tau_{1}$ of the short living component from constrained fits with $\tau_{2}=219 \mathrm{ps}$ (open squares); average positron lifetime $\tau_{a v}$ from constrained fits (full circles).

does not allow a definite identification of the positron state corresponding to the long living component of the spectrum. The two-component analysis could be extended down to RT by fixing the lifetime of the second component at $219 \mathrm{ps}$. The average positron lifetime $\tau_{a v}$ obtained from this constrained analysis is also presented in figure 2. A sigmoidal increase of $\tau_{a v}$ with the temperature is observed; special effects at the ferroelectric transition were not seen in this case. The room temperature value of $\tau_{a v}$ is $166 \pm 1 \mathrm{ps}$. This value is closer to the theoretical prediction [15] of 152 ps for free annihilation in bulk $\mathrm{BaTiO}_{3}$ than the experimental value of about $175 \mathrm{ps}$, measured at room temperature for sintered $\mathrm{BaTiO}_{3}$, after annealing in air at atmospheric pressure, by Massoud et al [14]. The moderate disagreement with the theoretical value may be due to a small unresolved contribution of trapping at thermally stable defects. On the other hand, it may be mentioned that the calculations tend to overestimate the positron annihilation rate with core electrons, leading to bulk lifetimes that can be $10 \%$ shorter than the experimental ones [23,24].

The temperature dependence of $\tau_{a v}$ strongly suggests a thermally activated process. A similar behaviour was also observed in sintered $\mathrm{BaTiO}_{3}$ by Massoud et al [14]. The possibility that the effect is to be explained in terms of competition trapping between shallow traps and vacancies was discussed and discarded in [14]. The argument was that the binding energy of positrons in a Rydberg state is expected to be very low in $\mathrm{BaTiO}_{3}$ due to the high dielectric constant of this material. An alternative interpretation can be based on the hypothesis of thermal formation of new traps. Based on this hypothesis, we have analysed our data in accordance to the simple two-state version of the well known positron-trapping model [25-27].

The trapping model can be used for obtaining the trapping rate at these sites from the measured average lifetimes $\tau_{a v}$, by means of the equation

$$
k_{v}=\tau_{b}^{-1} \frac{\tau_{a v}-\tau_{b}}{\tau_{b}-\tau_{a v}}
$$


where $\tau_{b}$ indicates the positron lifetime in the bulk crystal (straight line through the low temperature points in figure 2) and $\tau_{d}=\tau_{2}$ is the positron lifetime in the trap. According to the model, the trapping rate $k$ is proportional to the concentration of traps, and is thus expected to depend on the temperature as given by the relationship

$$
k_{v} \propto \exp \left(-H / k_{B} T\right)
$$

where $H$ is the trap formation enthalpy and $k_{B}$ the Boltzmann constant. This dependency is shown in the Arrhenius plot presented in figure 3, which corresponds to $H=0.50 \pm 0.01 \mathrm{eV}$. It is difficult to reconcile this value with the formation of intrinsic disorder in $\mathrm{BaTiO}_{3}$, which is estimated to require energies from $2.29 \mathrm{eV}$ to $2.58 \mathrm{eV}$ for various Schottky defects and $4.49 \mathrm{eV}$ to $7.56 \mathrm{eV}$ for different Frenkel pairs [2]. A possible alternatives is that the positron traps are formed by the dissociation of impurity-vacancy complexes, whose binding energies would fall in the correct interval [2]. We cannot discard, however, the hypothesis of a change of the charge state of impurity-related traps.

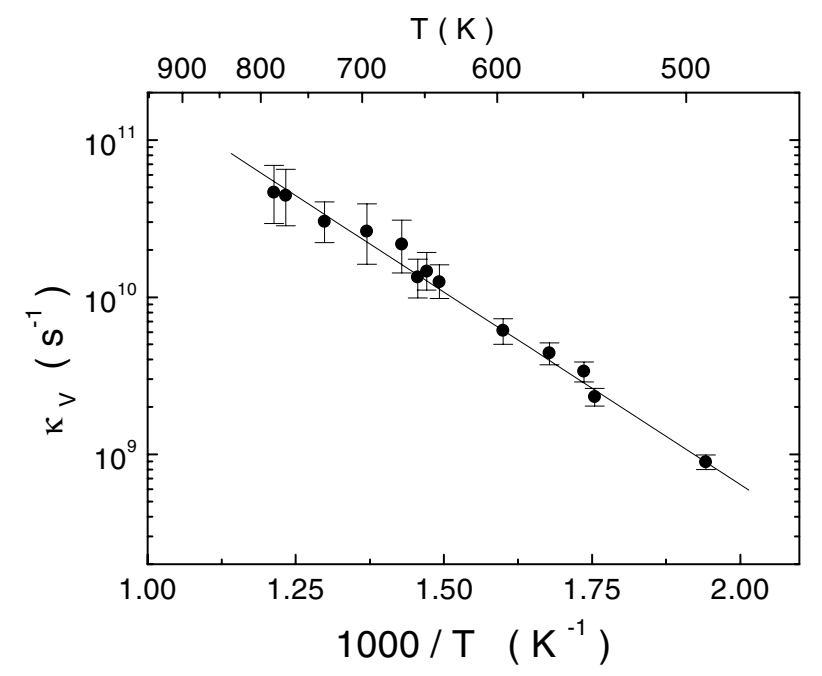

Figure 3. Arrhenius plot of the positron trapping rate in well annealed $\mathrm{BaTiO}_{3}$ single crystals, as obtained from the data of figure 2 according to the positron trapping model.

The conclusions of the present work can be summarized as follows:

(a) an irreversible behaviour of the temperature dependence of the positron lifetimes in asprepared $\mathrm{BaTiO}_{3}$ single crystals indicates positron trapping at vacancy-like defects;

(b) a reversible behaviour, consistent with the annealing of thermally unstable native defects, is obtained after heating in air at temperatures above $550 \mathrm{~K}$;

(c) different families of positron traps are present in as-prepared samples, which have tentatively been identified with $\mathrm{Ti}$ vacancies (negative charge state) and with $\mathrm{O}$ vacancies (charge state reversibly changing from positive to neutral and vice versa in dependence on the local Fermi level position);

(d) thermal activation of positron traps is observed in annealed samples, with an activation enthalpy which seems too low to be attributed to the formation of intrinsic disorder.

The present work gives evidence of the importance of native defects in determining positron annihilation characteristics in $\mathrm{BaTiO}_{3}$. Complexes formed by accidental impurities with compensating vacancies are also suspected to determine the thermal equilibrium behaviour 
with respect to positron trapping. Future applications of PAS as a technique for defect characterization in $\mathrm{BiTiO}_{3}$ require further preliminary studies on samples doped with controlled concentrations of impurities.

\section{References}

[1] Kittel C 1995 Introduction to Solid-State Physics 7th edn (New York: Wiley)

[2] Lewis G V and Catlow C R A 1986 J. Phys. Chem. Solids 4789

[3] Scharfschwerdt R, Mazur A, Schirmer O F, Hesse H and Mendricks S 1996 Phys. Rev. B 5415284

[4] Hehenkamp Th, Kurschat Th and Lühr-Tanck Wl 1986 J. Phys. F: Met. Phys. 16981

[5] Kluin J-E and Hehenkamp Th 1991 Phys. Rev. B 4411597

[6] Würschum R, Grupp C and Schaefer H-E 1995 Phys. Rev. Lett. 7597

[7] Kümmerle E A, Badura K, Sepiol B, Mehrer H and Schaefer H-E 1995 Phys. Rev. B 52 R6947

[8] Hautojärvi P and Corbel C 1993 Positron Spectroscopy of Solids ed A Dupasquier and A P Mills Jr (Amsterdam: IOP Press) p 491

[9] Sueoka O 1973 Phys. Lett. A 43459

[10] Troev T 1978 Phys. Lett. A 66233

[11] Tsuda N, Shirasaki S, Akahane T, Troev T and Chiba T 1979 Positron Annihilation ed R R Hasiguti and K Fujiwara (Japan Institute of Metals, Sendai) p 479

[12] Hsu F H and Hou T K 1992 Mater. Sci. Forum 105-110 655

[13] Süvegh K, Domján A, Tarsoly R and Vértes A 1996 J. Radioanal. Nucl. Chem. 211255

[14] Massoud A M, Krause-Rehberg R, Langhammer H T, Gebauer J and Mohsen M 2000 Mater. Sci. Forum 363-365 144

[15] Ghosh V J, Nielsen B and Friessnegg T 2000 Phys. Rev. B 61207

[16] Kirkegaard P, Pedersen N and Eldrup M 1989 PATFIT Program Ris $\phi$-M-2740 (RNL, Roskilde)

[17] Barathi A, Hariharan Y, Sood A K, Sankara Sastry V, Janawadkar M P and Sundar C S 1988 Europhys. Lett. 6 369

[18] Brusa R S, Grisenti R, Liu S, Oss S, Pilla O, Zecca A, Dupasquier A and Matacotta F A 1988 Physica C 15665

[19] Friessnegg T, Madhukar S, Nielsen B, Moodenbaugh A R, Aggarval S, Keeble D J, Pointdexter E H, Mascher P and Ramesh R 1999 Phys. Rev. B 5913365

[20] Barathi A, Sundar C S and Hariharan Y 1989 J. Phys.: Condens. Matter 11467

[21] McMullen T M, Jena P, Khanna S N, Li Yi and Jensen K O 1999 Phys. Rev. B 4310422

[22] Ishibashi S, Yamamoto R, Doyama M and Matsumoto T 1991 J. Phys.: Condens. Matter 39169

[23] Puska M J 1991 J. Phys.: Condens. Matter 33455

[24] Jensen K O 1989 J. Phys.: Condens. Matter 110595

[25] Brandt W 1967 Positron Annihilation ed A T Stewart and L O Roellig (New York: Academic) p 155

[26] Bergersen B and Stott M J 1967 Phys. Rev. Lett. 19307

[27] Connors D C and West R N 1969 Phys. Lett. A 3024 